\title{
Actinomycotic osteomyelitis of a long bone in an immunocompetent adult: a case report and literature review
}

\author{
Dong Jin Ryu', Yoon Sang Jeon ${ }^{1}$, Hea Yoon Kwon², Suk Jin Choi ${ }^{3}$, Tae Hoon Roh ${ }^{1}$ and Myung Ku Kim ${ }^{1 *}$
}

\begin{abstract}
Background: Actinomycosis is a rare, chronic granulomatous disease caused by Gram-positive anaerobic bacteria that colonize the oral cavity. Cervicofacial actinomycosis is the most frequent clinical presentation of actinomycosis, but hematogenous osteomyelitis at distant sites can occur in rare instance in immunocompromised or pediatric patients, only a few cases have been reported in healthy patients. Here we described a new case of distal femur osteomyelitis caused by Actinomyces in an adult patient who was immunocompetent and had no predisposing factors.
\end{abstract}

Case presentation: A woman aged 52 years with no history of trauma presented with severe pain, swelling, and increased local heat in the proximal area of the right knee 3 weeks after she first noticed discomfort. Magnetic resonance imaging showed persistent osteomyelitis of the distal metaphysis and diaphysis of the femur with a multifocal intraosseous abscess pocket. An incision and drainage of the abscess were conducted. The tissue culture, fungus culture, acid fast bacillus (AFB) culture, AFB smear, and tuberculosis polymerase chain reaction test results were negative. A pathologic examination confirmed the presence of actinomycosis. The patient was successfully treated with intravenous penicillin $\mathrm{G}$ for 8 weeks followed by oral amoxicillin-clavulanate for 6 weeks with repeated surgical debridement and drainage. After a 5-year follow up, the patient had no signs of recurring infection or complications and she had full range of movement in the affected knee.

Conclusions: Although rare, actinomycotic osteomyelitis can occur in healthy people. Furthermore, actinomycotic osteomyelitis is easily misdiagnosed as tuberculosis in areas with a high prevalence of tuberculosis. To detect and identify the bacteria accurately, pathologic examination should be performed as well as culture tests, because the probability for culture confirmation of actinomycosis is quite low. The initial treatment is vital to a successful outcome without ostectomy or amputation.

Keywords: Actinomyces, Osteomyelitis, Pathology, Culture

\section{Background}

Actinomyces is an anaerobic Gram-positive filamentous organism, commonly found at high concentrations in tonsillar crypts and gingivodental crevices. More than 30 species of Actinomyces have been described. Actinomyces israelii is the most prevalent species in human infections and is found in most actinomycosis [1]. Osteomyelitis caused by Actinomyces is uncommon, and located

\footnotetext{
* Correspondence: m9kim@inha.ac.kr

${ }^{1}$ Department of Orthopedic Surgery, College of Medicine, Inha University Hospital, 7-206, 3rd Street Sinheung-Dong, Jung-Gu, Incheon 400-103, South Korea

Full list of author information is available at the end of the article
}

primarily in the head, neck, and cervical areas [1, 2]. Actinomycotic osteomyelitis resulting from hematogenous seeding is rarely reported, and most cases occur in children or immunocompromised patients who have predisposing factors [1, 3-5]. However, a small number of cases of actinomycotic osteomyelitis in the lower legs of healthy people, especially the long bone, have been reported. It shows variable clinical presentation and leads to diagnostic confusion, can mimic malignancy, and result in delayed therapy. In particular, in areas with a high prevalence of tuberculosis, initial treatment may fail due to misdiagnosis and therefore incorrect treatment of tuberculosis [1, 6-9]. Here we described a new case of distal femur 
osteomyelitis caused by Actinomyces in an adult patient who was immunocompetent and had no predisposing factors.

\section{Case presentation}

A woman aged 52 years with no prior trauma presented with severe pain, swelling and increased local heat in the proximal area of the right knee. The patient's symptoms developed 3 weeks prior to her arrival at our hospital. She initially presented with pain in both knees at the local clinic and was treated with physical therapy and hyaluronic acid injections. The left knee pain resolved following treatment. However, the right knee pain persisted, and the patient reported that the pain and increased local heat had extended to the more proximal area. Furthermore, the patient developed a high fever of over $39^{\circ} \mathrm{C} 2$ weeks after first treatment. She was referred to our hospital with a suspected distal femur bony malignancy.

The patient had no past medical history of diabetes mellitus, hypertension, hepatitis, or systemic infection. The patient's Human Immunodeficiency Virus (HIV) test, and liver and kidney function tests were normal. Notably, she had a salphingectomy 15 years prior, and a single tooth extracted approximately 4 months prior to presentation. She received prophylactic antibiotics before the tooth extraction.

On the physical examination, an increased local heat in the proximal area of the right knee without an external wound, or draining sinus was confirmed. Body temperature was $38.8^{\circ} \mathrm{C}$. Laboratory test results showed the following: leukocytes $7260 / \mu \mathrm{L}$ (neutrophil 79.1\%), absolute neutrophil count 4050, C-reactive protein (CRP) $21.26 \mathrm{mg} / \mathrm{L}$, and erythrocyte sedimentation rate (ESR) $72 \mathrm{~mm} / \mathrm{h}$. We conducted synovial fluid analysis on the fluid extracted from the right knee joint. Synovial fluid analysis revealed a white blood cell count of 870/ $\mathrm{mm}^{3}$, a polymorphonuclear leukocyte of $45 \%$, and no crystals were found. Anteroposterior and lateral radiography of the right knee revealed multifocal osteolytic changes in the distal metaphysis area of the right femur. The lesion had an irregular margin but no sclerotic rim (Lodwick classification type 1B). There was no definite destruction of cortical bone. However, a subtle cortical thickening lesion suggesting solid type of periosteal reaction was found at superior aspect of lateral femoral condyle (arrow head, a) (Fig. 1). The axial (a), coronal (b),

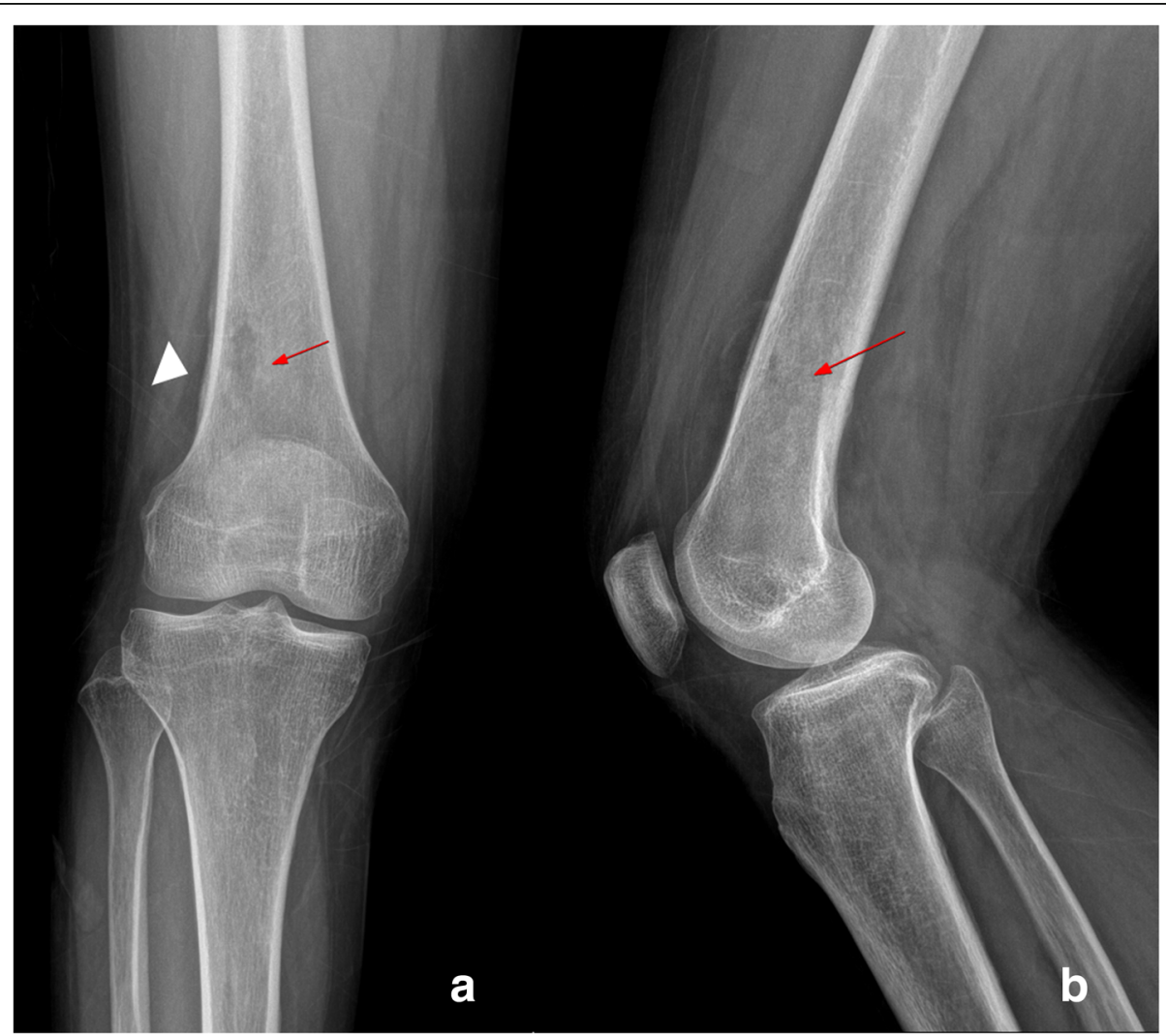

Fig. 1 Initial X-ray findings of the right knee. Anteroposterior (a) and lateral (b) radiography of the right knee revealed multifocal osteolytic changes (arrow) in the distal metaphysis area of the right femur. There was a geographic lesion with an irregular margin, but, no sclerotic rim (Lodwick classification type 1B). There was no destruction of cortical bone. However, a subtle cortical thickening lesion suggesting solid type of periosteal reaction was found at superior aspect of lateral femoral condyle (arrow head, a) 
and sagittal (c) T2-weighted magnetic resonance imaging (MRI) of the right knee, which were previously performed at another hospital (with lacking information of T1 weighted and enhanced image), are shown in Fig. 2. Multifocal intraosseous lesions $(6.1 \times 2 \times 2 \mathrm{~cm})$ at metaphysis and diaphysis of the distal femur were observed with no destruction of cortical bone. However, double-contour periosteal line, which suggesting periosteal reaction (arrow head, A and B) at lateral aspect, and marrow edema were found in the adjacent tissues (asterisk, B). Considering the patient's age, location of the lesion, margin, and absence of cortical destruction, we suspected osteomyelitis rather than a bony malignancy.

Still considering the possibility of malignancy, we made a $5 \mathrm{~cm}$ longitudinal incision in the anterolateral aspect of the distal femur. We split the vastus lateralis muscle and made an oval-shaped bony window in the lateral aspect of the femur. We evacuated yellowish pus-like intramedullary abscess, and the tissues were sent for Gram staining, routine culture, acid-fast bacillus (AFB) culture, fungus culture, and pathology test. Tissues were collected from various sites, and frozen pathology test was performed to exclude the possibility of malignancy. The emergency frozen pathology examination showed an absence of malignant cells. Continuous, massive marginal debridement and irrigation were performed. We inserted a negative-pressure drain into the intramedullary canal through the distal femur bony window. Intravenous first-generation cephalosporin (Cefazolin, $2 \mathrm{~g} / \mathrm{q} 8 \mathrm{hr}$ ) was empirically used to target methicillin-susceptible Staphylococcus aureus, which is still the most common cause of osteomyelitis. The tissue culture, fungus culture, AFB culture, AFB smear, and tuberculosis $(\mathrm{Tb})$ polymerase chain reaction (PCR) tests were negative.
Six days post-surgery, pathologic examination of a bone biopsy specimen revealed acute and chronic osteomyelitis. Furthermore, colonies of filamentous bacteria that were rimmed by neutrophils with sulfur granules, which are indicative of actinomycotic colonies, were present. (Fig. 3) However, we were unable to confirm the exact species of Actinomyces. As a result, the treatment plan was altered, and intravenous penicillin $G$ potassium (400 million IU/q6hr) was administered to target the actinomycotic osteomyelitis. The patient received repeated curettage and debridement on postoperative days 13, 27, 41 , and 54 because of continuous pus- like discharge through the suction drain and abnormal ESR and CRP results. The specimens obtained on days 13,27 , and 41 were still positive for sulfur granules on pathologic examination. However, the culture tests remained negative. The specimen obtained on day 54 was normal on following pathologic examination. At postoperative day 60 , the yellow pus-like discharge decreased in volume, and the ESR and CRP results were normal. The drain was removed, and the wound was closed. Penicillin G potassium was Intravenously administered for 8 weeks followed by amoxicillin-clavulanate taken orally at a dosage of $625 \mathrm{mg}$ every $6 \mathrm{~h}$ for 6 weeks. CRP and ESR were tested at 2-week intervals. After confirming the CRP and ESR values were normal for 3 consecutive intervals and that there were no signs of recurrence (local heating, pain, swelling, or painful range of motion), we discontinued the oral antibiotics. Antibiotics were discontinued after total 14 weeks of treatment.

Follow-up was performed at 6 months, 1 year, 3 years and 5 years after surgery. At the follow-up, X-rays showed that the bony window was not fully recovered, but there was no evidence of recurrence or pathological fracture (Fig. 4). During the 5-year follow-up period,

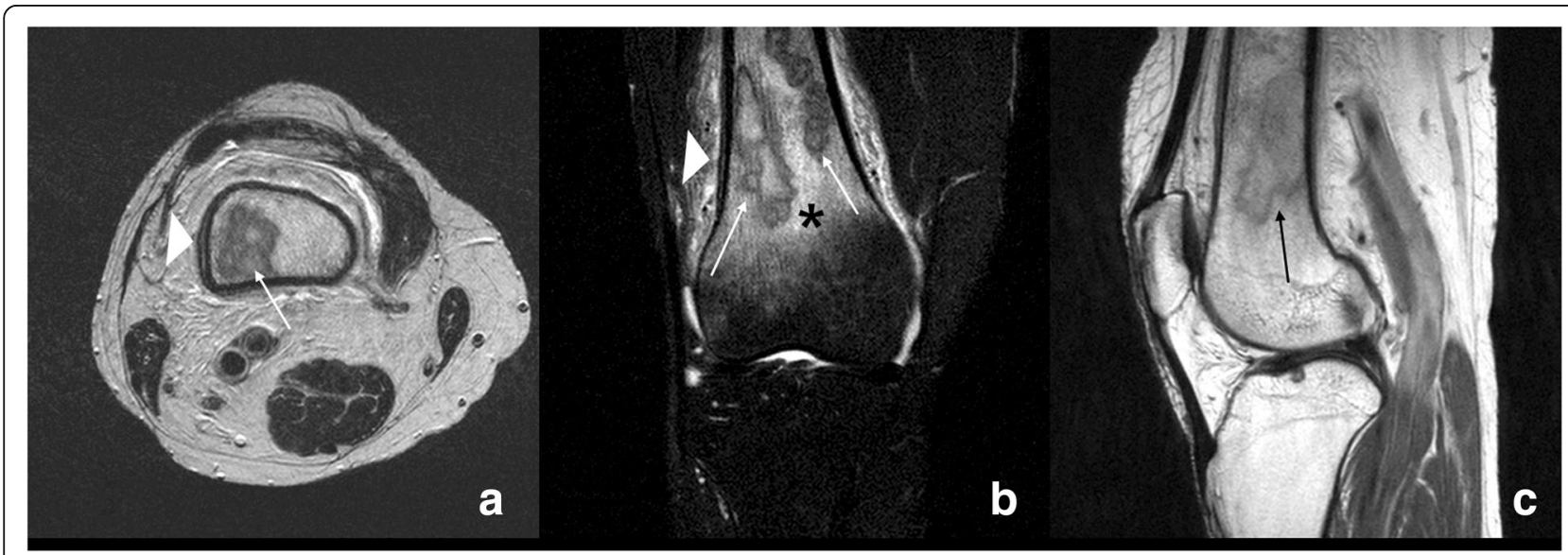

Fig. 2 Preoperative MRI findings of the right knee. Axial (a), coronal (b), and sagittal (c) T2-weighted MRls are shown. There were multifocal intraosseous lesions (arrows, $6.1 \times 2 \times 2 \mathrm{~cm}$ sized) at metaphysis and diaphysis of the distal femur. There was no destruction of cortical bone. However, double periosteal line, which suggesting periosteal reaction (arrow head, a and $\mathbf{b}$ ), and marrow edema were found in the surrounding tissues (asterisk, b) 


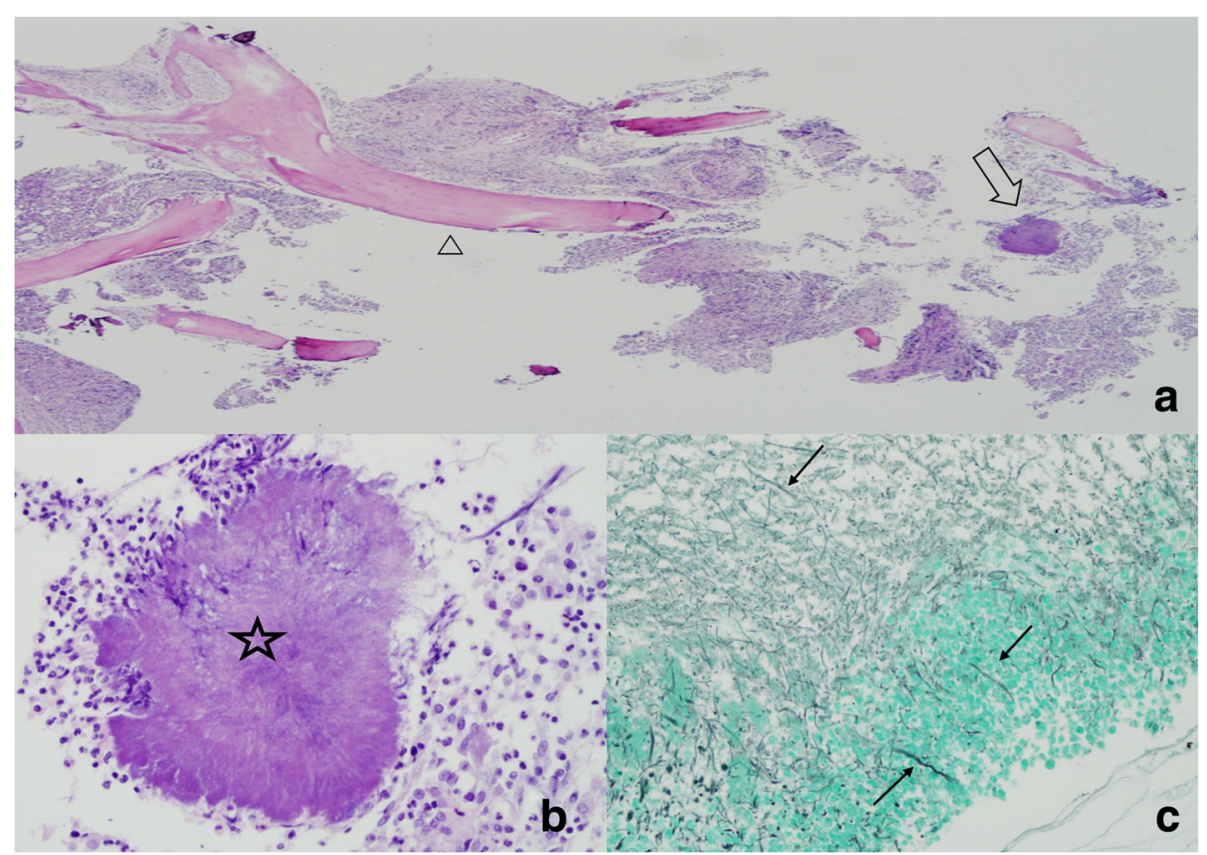

Fig. 3 Pathologic findings of drained materials. a The haematoxylin-eosin stain $(\times 4)$, of the curettage specimen shows necrotic bone detritus (arrowhead) and inflamed granulation tissue consistent with osteomyelitis. We identified a sulfur granule (arrow). b The haematoxylin-eosin stain $(\times 40)$, shows the sulfur granule (asterisk) is rimmed by neutrophils. The granule is composed of filamentous bacteria embedded in an amorphous matrix. c GMS stain $(\times 20)$ shows individual bacterial filaments (arrows)

CRP and ESR levels remained normal, and there were no symptoms of recurrence, such as local heating, swelling, and pain. In addition, the patient had full range of motion in the affected knee. The patient is satisfied with the result of treatment and enjoy daily life with no discomfort.

\section{Discussion and conclusions}

Between the years of 1990 and 2017, 8 cases of lower leg actinomycotic osteomyelitis have been published in the English-language literature (Table 1). Osteomyelitis of the long bone is generally attribute to hematogenous spread, trauma, or the presence of a prosthetic device [10]. Anaerobic osteomyelitis is usually rare [11], and typically reported in patients with a chronic underlying disease or following a complicated bone fracture. These cases usually present without hematogenous spread. Our patient had no specific infection source or predisposing disease other than a recent single-tooth extraction. Therefore, our presumption was that the tooth extraction resulted in hematogenous seeding of Actinomyces to a distant site, causing the osteomyelitis. There are only a few case reports of long bone osteomyelitis following an oral infection [12].

To diagnose an atypical osteomyelitis such as this case, malignancy (especially Ewing sarcoma) [13], metastatic tumor, Langerhans cell histiocytosis, and Garre's sclerosing osteomyelitis should be considered and excluded. The age of the patient, location of the lesion, clinical features, degree of progression, soft tissue reaction, existing sinus, and radiological findings (X-ray, CT, MRI, bone scan) should be considered together. In this case, because of the more suspecting osteomyelitis in the pre-exam, we started with two plans. First, biopsy would be conducted by a mini-open lateral approach, and when malignancy cells appeared in the frozen section, end the operation and prepare for the second bony malignancy operation. But in the actual operation field, there was definite pus-like abscess drainage and no cells suspected of malignancy in multiple frozen sections. After that, we conducted massive debridement and irrigation. However, because of the possibility of diagnostic errors in the emergency test through the frozen test [14], it would be more safe to confirm the biopsy exam and establish a final treatment plan.

Actinomycotic osteomyelitis can easily be mistaken for more common conditions with discharging sinuses, such as pyogenic osteomyelitis and tuberculosis [1], especially in areas with a high prevalence of tuberculosis. As a result, initial treatments may use an anti-tuberculosis agent due to a suspected misdiagnosis of tuberculosis osteomyelitis [1,9]. The incubation period until the symptoms are manifested after Actinomycosis infection has not been clearly established. It is known to be very diverse, and at literature review of current study show that it vary from three months [15] to as long as 15 years [9]. 


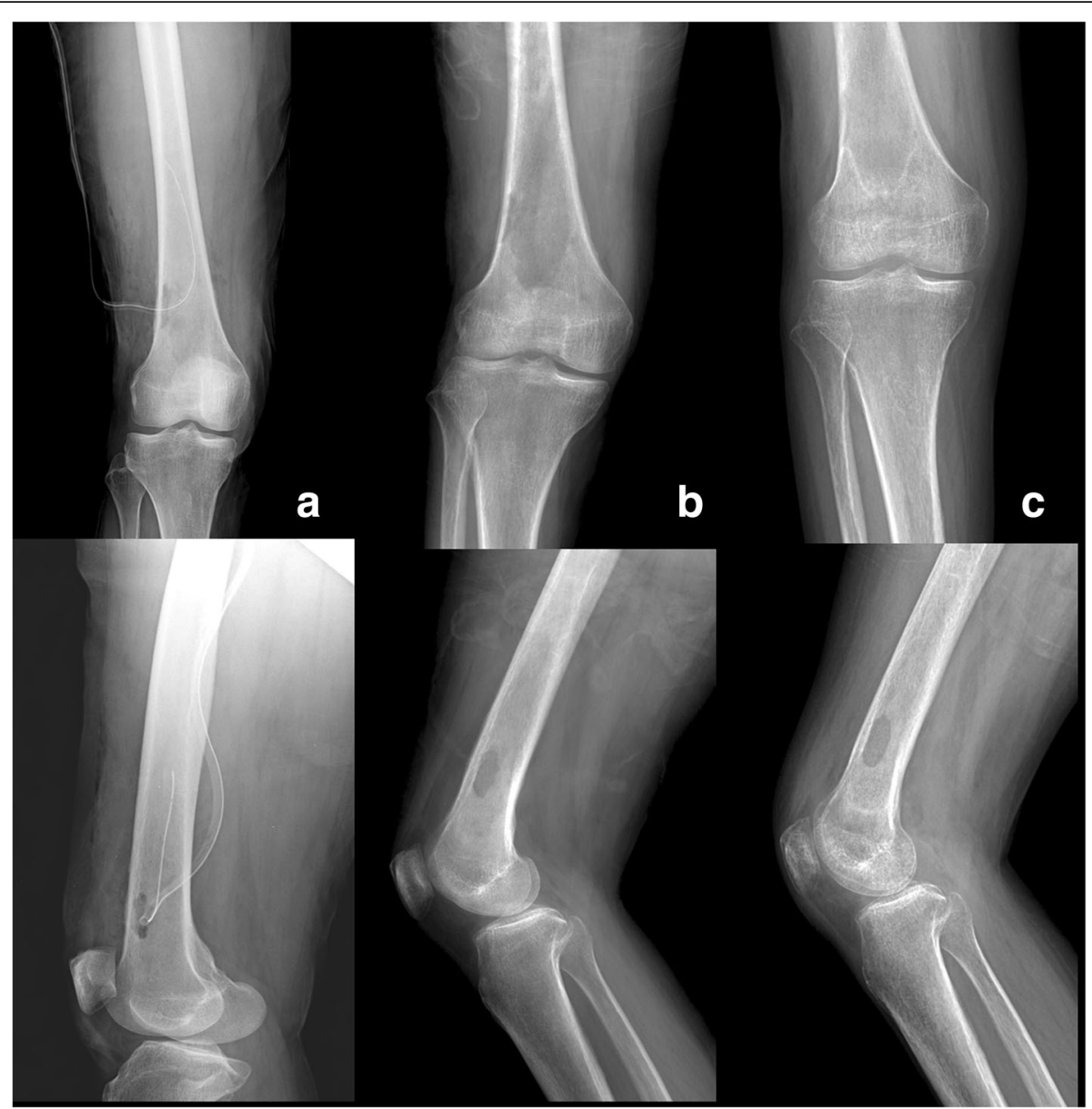

Fig. 4 Serial X-ray findings according to progress. a Post-operative anteroposterior and lateral radiography of the right knee shows the ovalshaped small bony window that was made at the lateral aspect of the distal femur and reveals the drain insertion status. b Anteroposterior and lateral radiography after the fifth debridement shows that the bony curettage range was wider. c Anteroposterior and lateral radiography at the 1 -year follow-up; the sclerotic rim is formed, and the bony window remains. There was no cortical destruction or periosteal reaction

Culture confirmation of the infectious organism is important for the development of an effective treatment strategy. Prompt transport of the specimens to the microbiology laboratory, preferably $\mathrm{n}$ anaerobic medial, is necessary for optimal isolation. However, Actinomyces is difficult to culture. Reiner et al. [16] could only confirmed 17 cases from a total of 35 suspected cases by culture. Thus, pathologic examination is vital to an accurate diagnosis. For our patient, the routine cultures, fungus culture, AFB culture, and Gram staining were negative. However, we were able to make the correct diagnosis by confirming the presence of sulfur granules on pathology examination. If there are atypical clinical findings or unexpected operative findings, we recommend culture, gram staining, $\mathrm{Tb}$ PCR, and pathologic examination. Early diagnosis and proper treatment for actinomycosis is important for a successful outcome and to prevent persistent draining sinus, deformation, sclerotic change of bone, and lower-limb amputation $[6,8,17]$.
Actinomyces is extremely susceptible to beta-lactams, especially penicillin $\mathrm{G}$ or amoxicillin, and drug resistance is not considered to be a problem. Patients require many times of drainage and debridement until the signs of infection are no longer present and the pathologic examination become negative. In addition, prolonged (at least 4 to 8 weeks) high doses of penicillin $\mathrm{G}$ or amoxicillin are needed. Notably, the antibiotic duration could likely be reduced for patients in whom optimal surgical resection of the infected tissue has been performed. Reiner et el. [16] suggested that surgical therapy was necessary in most instances. We did not use in our patient, Nayak et el. [17] reported that using bone cement (antibead) with a mixture of antibiotics can increase the local therapeutic effect. However, penicillin $G$ is not stable at the heat which generated during the process of making antibiotic cements. Therefore, amoxicillin would be a better choice for the antibiotic cement [18]. Vancomycin is also known to be effective antibiotics in Actinomyces 


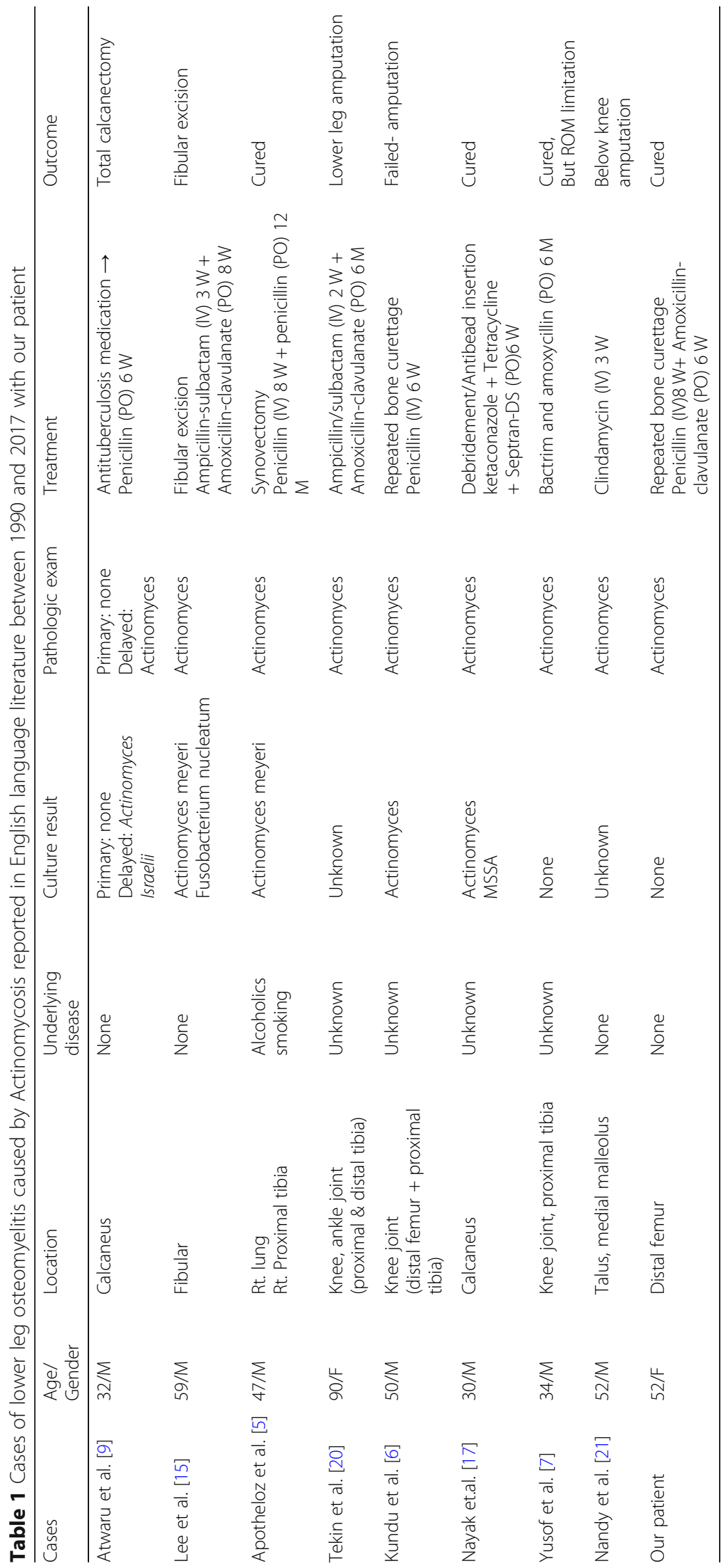


management [19]. In general, osteomyelitis due to Methicillin-susceptible Staphylococcus aureus or Methicillin-resistant Staphylococcus aureus is common, thus insertion of vancomycin loaded bone cement may be a good initial management until the final culture exam result.

Pending the results of culturing, a lesion with granules of branching Gram-positive bacteria should be empirically treated with penicillin and a sulfonamide. Actinomycotic osteomyelitis has a high rate of successful treatment outcomes, but several additional surgical treatments may be needed $[6,15]$. Kundu et al. suggested that a delayed diagnosis leads to sclerosis of the bone, which hampers the penetration of penicillin and makes infection control more difficult [6]. In the previously reported case, partial ostectomy or amputation was performed in 3 out of 8 patients (37.5\%) $[6,9,15$, 20]. Our patient was successfully treated with repeated marginal debridement and drainage without the need for ostectomy or amputation. The pus-like discharge in our patient persisted despite the IV antibiotics, open drainage, and wound care. Consequently, a total of five debridement and bone curettages were performed and the duration of antibiotic usage was quite long, with 8 weeks for intravenous antibiotics and 6 weeks for oral antibiotics.

Actinomycosis is an extremely rare and challenging disease for orthopedic surgeons and can be easily mistaken for more common conditions with discharging sinuses, such as pyogenic osteomyelitis and tuberculosis. This is especially common in areas with a high prevalence of tuberculosis. Routine culture and Gram staining are still important, but the culture confirmation rate is low. Therefore, the lessons learned from this case are that it is important to make an accurate diagnosis early on by carrying out the procedure in accordance with various exam (including pathology, Tb PCR, culture test), even if it is a typical or atypical lesion. With a prompt diagnosis and initial treatment, actinomycotic osteomyelitis can be successfully treated without ostectomy or amputation.

\section{Abbreviations \\ AFB: Acid-fast bacillus; IV: Intravenous; PCR: Polymerase chain reaction; ROM: Range of motion; Tb: Tuberculosis}

\section{Acknowledgements}

Not applicable.

\section{Funding}

This work was supported by INHA UNIVERSITY HOSPITAL Research Grant. The funding body had no influence in the design of the study and collection, analysis, and interpretation of data and in writing the manuscript.

Availability of data and materials

Data are contained within the manuscript.

\section{Authors' contributions}

DJR wrote the manuscript including the literature review. YSJ had substantial contributions to conception and design. HYK and SJC contributed with the conception of the work, interpreted laboratorial data and revised the images. THR had been involved in revising the manuscript critically. MKK is an experienced knee surgeon, had drafted the work and critically revised the manuscript. All authors have read and approved the final manuscript and agreed both to be personally accountable for the author's own contributions and to ensure that questions related to the accuracy or integrity of any part of the work, even ones in which the author was not personally involved, are appropriately investigated, resolved, and the resolution documented in the literature.

\section{Ethics approval and consent to participate}

Not applicable.

\section{Consent for publication}

We obtained written informed consent from the patient for publication of this case report and any accompanying images and data. A copy of the written consent is available for review by the editor of this journal.

\section{Competing interests}

The authors declare that they have no competing interests.

\section{Publisher's Note}

Springer Nature remains neutral with regard to jurisdictional claims in published maps and institutional affiliations.

\section{Author details}

'Department of Orthopedic Surgery, College of Medicine, Inha University Hospital, 7-206, 3rd Street Sinheung-Dong, Jung-Gu, Incheon 400-103, South Korea. ${ }^{2}$ Department of Infectious Disease, College of Medicine, Inha University Hospital, Incheon, South Korea. ${ }^{3}$ Department of Pathology, College of Medicine, Inha University Hospital, Incheon, South Korea.

Received: 12 September 2018 Accepted: 15 April 2019

Published online: 01 May 2019

\section{References}

1. Ferry T, Valour F, Karsenty J, Breton P, Gleizal A, Braun E, et al. Actinomycosis: etiology, clinical features, diagnosis, treatment, and management. Infect Drug Resist. 2014:183.

2. Sezer B, Akdeniz B, Günbay S, Hilmioğlu-Polat S, Başdemir G. Actinomycosis osteomyelitis of the jaws: report of four cases and a review of the literature. J Dent Sci. 2017;12:301-7.

3. Catalano-Pons C, Raymond J, Chalumeau M, Armengaud J, Kalifa G, Gendrel D. Case 2: Paediatric chronic osteomyelitis: report of two cases. Acta Paediatr. 2007;96:1849-52

4. Robinson JL, Vaudry WL, Dobrovolsky W. Actinomycosis presenting as osteomyelitis in the pediatric population. Pediatr Infect Dis J. 2005;24:365-9.

5. Apothéloz C, Regamey C. Disseminated infection due to Actinomyces meyeri: case report and review. Clin Infect Dis. 1996;22:621-5.

6. Kundu ZS, Bhardwaj G, Sangwan SS, Arora B. Actinomycosis of the knee: a case report. J Orthopaed Traumatol. 2003;4:133-5.

7. Yusof MI, Yusof AH, Salleh MSM, Harun A. Actinomycosis of the knee. Malays J Med Sci. 2005;12:68-9.

8. Vandevelde AG, Jenkins SG, Hardy PR. Sclerosing osteomyelitis and Actinomyces naeslundii infection of surrounding tissues. Clin Infect Dis. 1995;20:1037-9.

9. Atwaru RM. Actinomycosis of the calcaneus - a case report. SA Ortho J. 2009:59-62.

10. Brook I, Frazier EH. Anaerobic osteomyelitis and arthritis in a military hospital: a 10-year experience. Am J Med. 1993;94(1):21-8.

11. Walter $G$, Vernier $M$, Pinelli $P O$, Million M, Coulange M, Seng P, et al. Bone and joint infections due to anaerobic bacteria: an analysis of 61 cases and review of the literature. Eur J Clin Microbiol Infect Dis. 2014;33:1355-64.

12. Germann $\mathrm{G}$, et al. Hematogenous osteomyelitis of the hand skeleton in adults after dental maxillary infections. Ann Plast Surg. 1996;37(1):106-10.

13. Henninger B, Glodny B, Rudisch A, Trieb T, Loizides A, Putzer D, et al. Ewing sarcoma versus osteomyelitis: differential diagnosis with magnetic resonance imaging. Skelet Radiol. 2013;42:1097-104. 
14. Bauer TW, Slaw RJ, McKenney JK, Patil DT. Validation of whole slide imaging for frozen section diagnosis in surgical pathology. J Pathol Inform. 2015;6:49.

15. Lee MJ, Ha YE, Park HY, Lee JH, Lee YJ, Sung KS, et al. Osteomyelitis of a long bone due to fusobacterium nucleatum and Actinomyces meyeriin an immunocompetent adult: a case report and literature review. BMC Infect Dis. 2012;12.

16. Reiner SL, Harrelson JM, Miller SE, Hill GB, Gallis HA. Primary actinomycosis of an extremity: a case report and review. Rev Infect Dis. 1987;9:581-9.

17. Nayak AR, Kulkarni S, Khodnapur GP, Santosh V. Actinomycotic fungal infection of foot - a case report. Int J Biomed Advance Res. 2013;4:946-9.

18. Soares D, Leite P, Barreira P, Aido R, Sousa R. Antibiotic-loaded bone cement in total joint arthroplasty. Acta Orthop Belg. 2015;81:184-90.

19. Steininger $C$, Willinger $B$. Resistance patterns in clinical isolates of pathogenic Actinomyces species. J Antimicrob Chemother. 2016;71:422-7.

20. Tekin R, Gem M, Karakaya YA. Primary Actinomycosis of the knee and ankle joint: an unusual manifestation. J Bacteriol Parasitol. 2012;03.

21. Bettesworth J, Gill K, Shah J. Primary Actinomycosis of the foot: a case report and literature review. J Am Col Certif Wound Spec. 2009;1:95-100

Ready to submit your research? Choose BMC and benefit from:

- fast, convenient online submission

- thorough peer review by experienced researchers in your field

- rapid publication on acceptance

- support for research data, including large and complex data types

- gold Open Access which fosters wider collaboration and increased citations

- maximum visibility for your research: over $100 \mathrm{M}$ website views per year

At BMC, research is always in progress.

Learn more biomedcentral.com/submissions 